\title{
Green Synthesis of Iron Nanoparticles using Aqueous Extract of Musa ornata Flower Sheath against Pathogenic Bacteria
}

\author{
S. SARANYA*, K. VIJAYARANI AND S. PAVITHRA \\ Department of Animal Biotechnology, Madras Veterinary College, Tamil Nadu Veterinary and Animal Sciences University, \\ Chennai-600 007, India
}

Saranya, et al.: Synthesis of Iron Nanoparticles against Pathogenic Bacteria

\begin{abstract}
Iron nanoparticles were synthesized using Musa ornata flower sheath extract. The optimum precursor salt concentration, $\mathrm{pH}$ of the reaction mixture, ratio between reducing agent and precursor salt and time for the synthesis of iron nanoparticles were found to be $5 \mathrm{mM}, 9.0,3: 7$ and $0^{\text {th }} h$, respectively. The synthesized iron nanoparticles were characterised by $\mathrm{UV} / \mathrm{Vis}$ absorption spectroscopy, Fourier transform infrared spectroscopy, X-ray diffraction spectroscopy, atomic force microscope and particle size analyser. UV/Vis absorption showed a characteristic absorption peak of iron oxide nanoparticles in the range of 250-350 $\mathrm{nm}$. Fourier transform infrared spectroscopy measurement was carried out to identify the possible molecules like carbonyl, $\mathrm{CH}$ and $\mathrm{OH}$ band. From the $\mathrm{X}$-ray diffraction method, it was found that the average particle size of magnetite nanoparticles was found to be $43.69 \mathrm{~nm}$. The synthesized iron nanoparticles had antibacterial activity against pathogenic bacteria like Staphylococcus aureus, Streptococcus agalactiae, Escherichia coli and Salmonella enterica by well diffusion method. This biosynthesis approach has been found to be cost effective, eco-friendly and promising for applications in various fields.
\end{abstract}

Key words: Iron nanoparticles, Musa ornata, XRD, FTIR, AFM, antibacterial activity

Nanotechnology, with a wide range of applications in biology, medicine and engineering, is a rapidly growing field ${ }^{[1]}$. Nanotechnology is concerned with the synthesis of nanoparticles of various sizes and shapes and their potential applications ${ }^{[2,3]}$. Though synthesis of nanoparticles by physical and chemical methods offers well distinct and pure nanoparticles, these nanoparticles are quite expensive and may not be environment friendly ${ }^{[4-6]}$. Use of microorganisms and plant materials could be an alternative to the physical and chemical synthesis of nanoparticles. The vision of utilizing the natural resources for the synthesis of metal nanoparticles has turned out to be a competent and environmentally safe approach. Green synthesis of metal nanoparticles using extracts of plant components is considered to be less expensive and environment friendly. During the green synthesis of metallic nanoparticles, the polyol components present in the plant extracts are responsible for the bioreduction of metal ions whereas water soluble heterocyclic components stabilize the nanoparticles formed ${ }^{[7,8]}$.
Metal nanoparticles are reported to have magnetic, catalytic, optical, antiinflammatory and antimicrobial properties. Among these properties antimicrobial property is considered to be one of the important property, which might have lot of potential in animal and human medicine. Apart from being eco-friendly and less expensive, plant extract mediated nanoparticles are reported to have extensive antimicrobial activity.

In the present study, iron nanoparticles (FeNPs) were synthesized using a rapid, single step, green biosynthetic method employing aqueous extract of Musa ornata (banana) flower sheath as both the reducing and capping agent. Further, the synthesised nanoparticles were tested for antimicrobial activity against pathogenic organisms.

This is an open access article distributed under the terms of the Creative Commons Attribution-NonCommercial-ShareAlike 3.0 License, which allows others to remix, tweak, and build upon the work non-commercially, as long as the author is credited and the new creations are licensed under the identical terms

Accepted 03 July 2017

Revised 31 January 2017

Received 15 October 2016

Indian J Pharm Sci 2017;79(5):688-694 


\section{MATERIALS AND METHODS}

Ferrous sulphate $\left(\mathrm{FeSO}_{4}\right)$, sodium hydroxide $(\mathrm{NaOH})$ and hydrochloric acid (HCL) was purchased from Merck, India Ltd. All the chemicals were in analytical grade and used without any further purification. Streptococcus agalactiae (ATCC 13813), Staphylococcus aureus (ATCC BAA 976), Salmonella enterica (ATCC 13076), and Esherichia coli (ATCC 43888) strains were purchased from American type culture collection, USA and used in the study. Nutrient agar and MacConkey agar was purchased from Himedia Pvt. Ltd., India. M. ornata flower was purchased from a local retail shop in Chennai and utilized for the preparation of flower sheath extract.

\section{Preparation of $M$. ornata flower sheath extract:}

M. ornata flower sheath was collected and washed thoroughly in water to remove the dust and other particulate matter. Fine pieces of sheath in water $(200 \mathrm{~g} / \mathrm{l})$ were heated at $60^{\circ}$ for $20 \mathrm{~min}$ followed by filtering through filter paper to separate out the extract. The extract was stored at $4^{\circ}$ for further experiments.

\section{Synthesis of FeNPs and effect of various parameters on the synthesis of FeNPs:}

A solution of $\mathrm{FeSO}_{4}$ (1 to $5 \mathrm{mM}$ ) was prepared by dissolving the $\mathrm{FeSO}_{4}$ in distilled water. In a typical green synthesis protocol, M. ornata flower sheath extract was used to reduce and cap the Fe ions. $10 \mathrm{ml}$ of sheath extract was added into the aqueous solution of $\mathrm{FeSO}_{4}$ with normal atmospheric pressure. The mixture was constantly stirred at $70-80^{\circ}$ for $8 \mathrm{~h}$ and subjected to further stirring at room temperature overnight without heating. The effects of different concentrations of precursor salt solution ( 1 to $5 \mathrm{mM}), \mathrm{pH}$ and time $(0,2,4,6,8,24 \mathrm{~h})$ on the stability of FeNPs were studied by UV/Vis spectrometry. The $\mathrm{pH}$ of reaction mixture was adjusted with $0.1 \mathrm{~N} \mathrm{HCL}$ and $0.1 \mathrm{M} \mathrm{NaOH}$ solution ( $\mathrm{pH} 4,5,6,7,8,9$ and 10) using calibrated $\mathrm{pH}$ meter. To check whether the ratio between reducing agent and precursor salt solution have any effect on the rate and shape of nanoparticles formation, the reaction mixtures were prepared in different ratios $(1: 9,2: 8,3: 7$, 4:6 and 5:5) and the maximum absorption wavelengths of iron colloids were recorded in UV/Vis region (from $200 \mathrm{~nm}$ to $800 \mathrm{~nm}$ ).

\section{UV/Vis spectrophotometric analysis of FeNPs:}

Synthesis of FeNPs was preliminarily confirmed by recording the absorbance in UV/Vis spectra at a range of 200-800 nm. Change in surface plasmon resonance
(SPR) of nanoparticles in the dispersion was recorded using UV/Vis spectrophotometer.

X-Ray Diffraction (XRD) and Fourier transform infra-red spectrophotometric (FTIR) analysis of FeNPs:

FeNPs in the powder form were used for XRD and FTIR spectroscopy analysis. The XRD patterns were collected on Bruker AXS D8 Advanced X-ray diffractometer with $\mathrm{Cu} \mathrm{K} \alpha$ radiation and scanning angle $2 \theta$ over the range of $10-80^{\circ}$. XRD patterns were

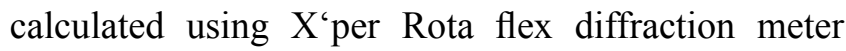
using $\mathrm{Cu} \mathrm{K}$ radiation and $\lambda=1.5406 \AA$. Crystallite size was calculated using Scherrer Eqn., $\mathrm{CS}=\mathrm{K} \lambda / \beta \cos \theta$, where $C S$ is the crystallite size, constant $K=0.94$, $\beta$ is the full width at half maximum (FWHM) $(\beta=\mathrm{FWHM}$ $\mathrm{x} \pi / 180), \lambda=1.5406 \times 10-10$ and $\cos \theta=$ Bragg angle ${ }^{[9]}$. FTIR was used to characterize the nanoparticles using the powdered sample by $\mathrm{KBr}$ pellet technique in the range of $400-4000 \mathrm{~cm}^{-1}$.

Atomic force microscope (AFM), Zeta potential and particle size analysis of FeNPs:

Synthesized FeNPs were characterized by AFM for their size, morphology and agglomeration. AFM image was taken with silicon cantilevers with contact mode. The mean particle size diameter and polydispersity indices of synthesized FeNPs were measured using photon correlation spectroscopy. The stability of nanoparticles in a colloidal suspension was determined based on the zeta potential value.

\section{Antibacterial activity of FeNPs:}

FeNPs synthesized using $M$. ornata flower sheath extract were tested for antibacterial activity by well diffusion method against $S$. agalactiae, $S$. aureus, $S$. enterica and E. coli. Twenty four hour fresh cultures of the bacterial pathogens were prepared on agar plates and the standardized (McFarland No.0.5) inoculums were used for the antibacterial assay. Powdered FeNPs were dissolved in sterile distilled water and sonicated. Four wells each of $5 \mathrm{~mm}$ diameter were made on each plate and the synthesized FeNPs solution, at a concentration of 1 to $4 \mathrm{mg} / \mathrm{ml}$ was loaded in each well. The plates were then incubated at $37^{\circ}$ for $24 \mathrm{~h}$ and the zone of inhibition was measured.

\section{RESULTS AND DISCUSSION}

Nanoparticulate technology has been found to have a wide range of applications since most of the biological processes occur at nanoscale levels. 
Chemical synthesis of nanoparticles involves the use of chemical reductants and the precursor salts in varying ratios. Establishing environment friendly nanoparticulate technology will be a welcome step in the modern scientific era. Green synthesis of metallic nanoparticles using plant extracts as reducing and capping agents is considered as a welcome step towards achieving eco-friendly and cheaper methods of generating nanoparticles. Moreover, green synthesis is gaining importance due to easy availability of plant materials and their pharmacological significance. Few metallic nanoparticles like $\mathrm{MgO}, \mathrm{CaO}$ and $\mathrm{ZnO}$ have been reported to have antibacterial activities ${ }^{[10]}$, which has prompted studies to assess the utility of metallic nanoparticles for their antibacterial activities. In present study, the flower sheath extract of M. ornata was used to synthesize FeNPs.

Synthesis of FeNPs was confirmed by the characteristic change of the sheath extract and aqueous $\mathrm{FeSO}_{4}$ mixture colour to dark yellowish brown. This indicated the reduction of aqueous iron ions to FeNPs when added to flower sheath extract of $M$. ornata and the colour reaction is the result of excitation of surface plasmon vibration in the metal nanoparticles ${ }^{[11]}$.

The bio reduction of $\mathrm{Fe}$ ions in aqueous solutions was monitored by measuring UV/Vis spectra. UV/Vis spectral analysis was done at a wavelength range of 200-800 $\mathrm{nm}$ to study the absorption spectra of green synthesized FeNPs and the absorption peaks were observed at 250-350 $\mathrm{nm}$ ranges due to the excitation of surface plasmon vibrations in FeNPs as has been reported earlier ${ }^{[12]}$. Effect of precursor salt solution on nanoparticles synthesis revealed that $5 \mathrm{mM}$ concentration of $\mathrm{FeSO}_{4}$ resulted in maximum nanoparticles synthesis with the absorption peak around $310 \mathrm{~nm}$ (fig. 1). The $\mathrm{pH}$ of the medium plays an important role in the size variability and dissolution kinetics of the nanoparticles ${ }^{[13]}$. As $\mathrm{pH}$ has been found to affect the stability of nanoparticles as well, in our study, the solution was adjusted to different $\mathrm{pH}$ and the concentration of $\mathrm{FeSO}_{4}$ was kept at $5 \mathrm{mM}$ in 1:1 ratio. Maximum absorbance peak of FeNPs formation was found at $\mathrm{pH} 9.0$ (fig. 2). As observed during the synthesis of silver nanoparticles ${ }^{[14]}$, probably because of the availability of large number of functional groups for binding, more number of FeNPs were formed at $\mathrm{pH}$ 9.0. In general, alkaline $\mathrm{pH}$ was found to be optimum for the synthesis of metallic nanoparticles. The ratio between reducing and precursor salt solution implies the formation of nanoparticles and it varies

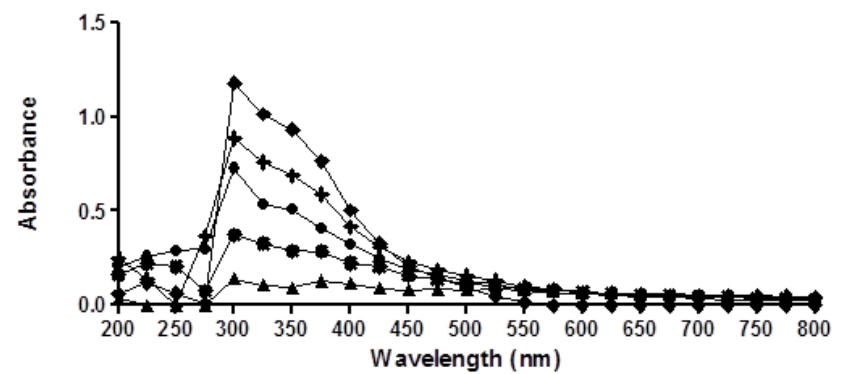

Fig. 1: Effect of $\mathrm{FeSO}_{4}$ precursor concentration on FeNPs synthesis

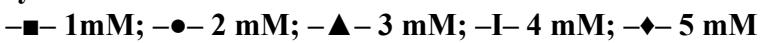

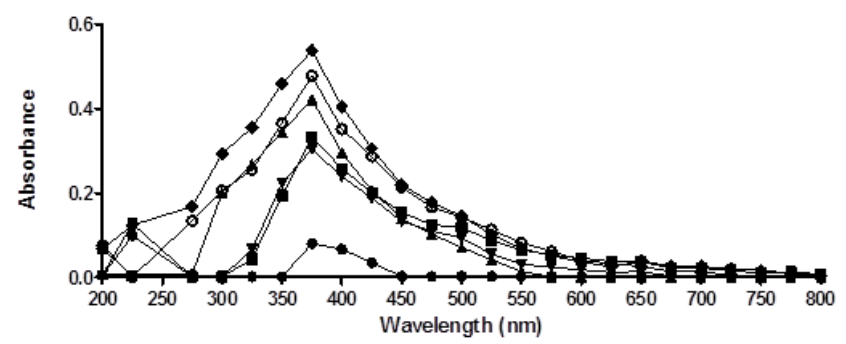

Fig. 2: Effect of pH on FeNPs synthesis

$-\bullet-\mathrm{pH}=5 ;-\square-\mathrm{pH}=6 ;-\Delta-\mathrm{pH}=7 ;-\nabla-\mathrm{pH}=8 ;-\bullet-\mathrm{pH}=9 ;-\sigma-$ $\mathrm{pH}=10$

from plant to plant. In the present study, maximum nanoparticle synthesis was achieved in the ratio of 3:7 (fig. 3). Though the reaction time is said to affect the stability of nanoparticles, we found no significant difference as far as reaction time is concerned after the addition of reducing agent (fig. 4).

The XRD technique was used to assess the composition and phase of the nanoparticles generated. Characteristics peak for nano iron have been observed at $2 \theta=36.4^{\circ}$ (fig. 5). This broadened diffraction peak suggested that the resultant nanoparticles are crystalline in nature with a size around $43.69 \mathrm{~nm}$ as calculated by Scherrer equation. FTIR spectra of $M$. ornata leaf extract are shown in fig. 6A. FTIR is used to study the surface interaction between synthesized nanoparticles with other molecules involved in the synthesis and stabilization of the nanoparticles ${ }^{[13]}$. The major absorption peaks in FTIR spectra of M. ornata leaf extract were mainly located at $3254.05,1635.17$, $525.09,474.54$ and $419.00 \mathrm{~cm}^{-1}$. The presence of peak at $3254.05 \mathrm{~cm}^{-1}$ indicate the possible O-H stretching vibration of phenol groups, which might be responsible for the formation and stabilization of nanoparticles ${ }^{[15]}$.

The FTIR spectrum of synthesized FeNPs displayed three strong bands around 3383.42, 1634.15 and $480.69 \mathrm{~cm}^{-1}$ (fig. 6B). Kumar and Singhal have reported the presence of similar bands at 472, 1634 


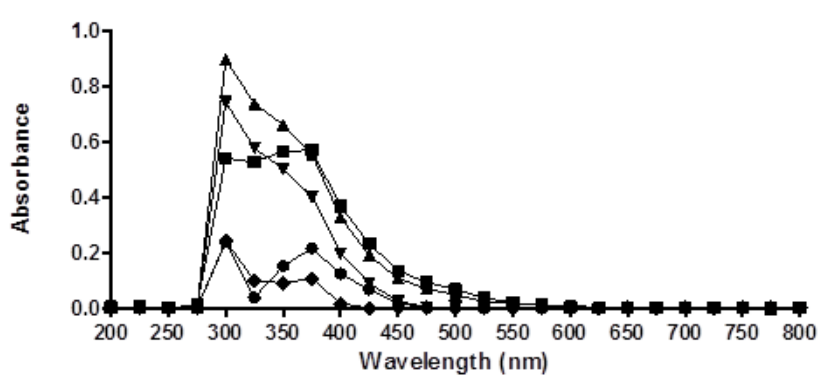

Fig. 3: Effect of ratio of reducing agent to precursor concentration on FeNPs synthesis

$-\bullet-1: 9 ;-\bullet-2: 8 ;-\Delta-3: 7 ;-\nabla-4: 6 ;-\downarrow-5: 5$

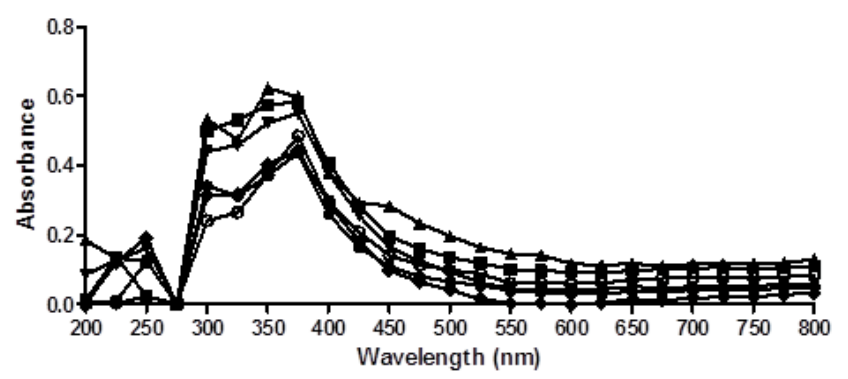

Fig. 4: Effect of reaction time on FeNPs synthesis

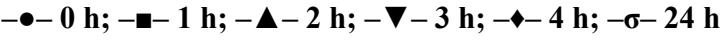

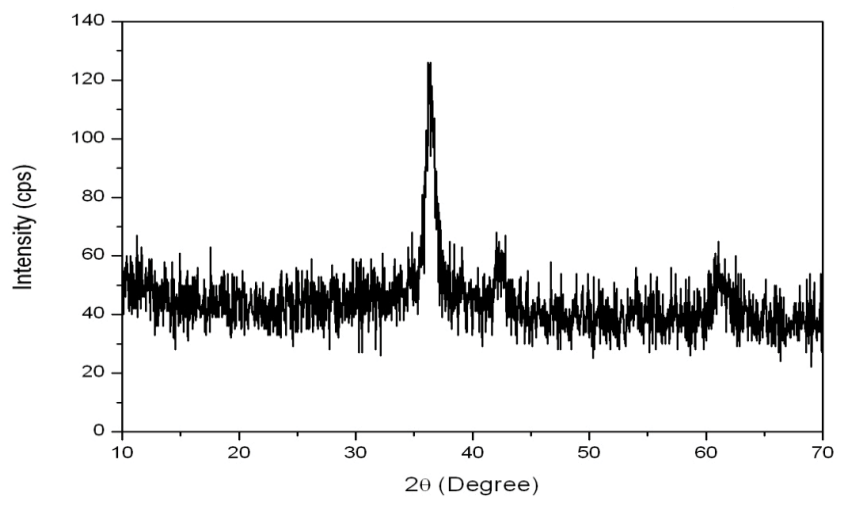

Fig. 5: X-ray diffraction pattern of FeNPs

Characteristics peak for nano iron have been observed at $2 \theta=36.4^{\circ}$

and $3438 \mathrm{~cm}^{-1}$ when colloidal $\beta-\mathrm{Fe}_{2} \mathrm{O}_{3}$ was synthesized in the presence of varying $\mathrm{Co}^{2+}$ amount ${ }^{[16]}$. Based on our study, the vibration bands could be assigned as $529.92 \mathrm{~cm}^{-1}(\mathrm{Fe}), \quad 1634.15 \mathrm{~cm}^{-1} \quad\left(\mathrm{H}_{2} \mathrm{O}\right.$ bending vibration) and a broad peak at $3383.42 \mathrm{~cm}^{-1}$. Presence of organic molecule on the surface of Fe NPs has been reported to have an influence on the FTIR peaks ${ }^{[17]}$ and the broad peak observed around $529.92 \mathrm{~cm}^{-1}$ instead of two sharp peaks, might be due to the organic molecule from the leaf extract on the surface of FeNPs. The weak band at $3837.93 \mathrm{~cm}^{-1}$ could well be attributed to the unsaturated nitrogen $(\mathrm{C}-\mathrm{N})$ compounds from the leaf extract.

The AFM image of FeNPs has been presented as fig. 7 . AFM topographic three dimensional images indicated the physically dispersed FeNPs. The magnetite nanoparticles were found to be oriented in one direction due to the magnetic properties. The surface potential of FeNPs was determined by zeta potential analyser, which is an essential characterization tool to assess the stability of the nanoparticles in aqueous solution. The theoretical limit of stability of particles in a colloidal system is $30 \mathrm{mV}^{[18]}$ and FeNPs synthesized by us had a zeta potential value of $\pm 35 \mathrm{mV}$ indicating that the synthesized FeNPs had moderate stability (fig. 8).

Particle size and size distribution of the FeNPs were further determined with the photon correlation spectrometer. Fig. 9 depicts a normal particle size distribution (PSD) of the synthesized iron particles with respect to the diameter of the FeNPs. The frequency distribution (on Y-axis) was on weight basis. The average particle size ( $\mathrm{z}$ - average) was found to be $86.2 \mathrm{~nm}$ and its polydispersity index was 0.305 . The zeta potential and particle size analysis revealed that the synthesized FeNPs had moderate stability in colloidal suspension. For that reason the synthesized FeNPs had the average size of $43.69 \mathrm{~nm}$ (powder form) in XRD analysis. Whereas in case of particle size analysis, the synthesized FeNPs had an average particle size of $86.2 \mathrm{~nm}$, which might be due to the aggregation of FeNPs in a colloidal suspension.

With ever increasing concerns over the use of antibiotics, look out for alternatives is on the rise. Molecules which do not lead to resistance are the most sought ones. Antimicrobial properties of metallic nanoparticles against certain pathogenic organisms have been reported. The antimicrobial activity of FeNPs synthesized using $M$. ornate flower sheath extract was investigated against most common foodborne pathogens and multi drug resistant bacteria like S. agalactiae, $S$. aureus, E. coli and $S$. enterica by well diffusion method. Though the FeNPs had a definite antibacterial activity against all the tested bacteria, no significant differences were noticed between the different pathogens except in case of $S$. enterica (fig. 10). The FeNPs did not have appreciable antibacterial activity at $5 \mathrm{mg} / \mathrm{ml}$ concentration compared to 10,15 and $20 \mathrm{mg}$ concentrations. The FeNPs synthesized by $M$. ornate flower sheath extract was found to have highest antimicrobial activity against S. aureus $(32 \mathrm{~mm})$ and $S$. agalactiae $(28 \mathrm{~mm})$.

It is concluded that, green synthesis of FeNPs using $M$. ornate flower sheath extract was successful at a higher $\mathrm{pH}$ of 9.0 without using any toxic chemicals 


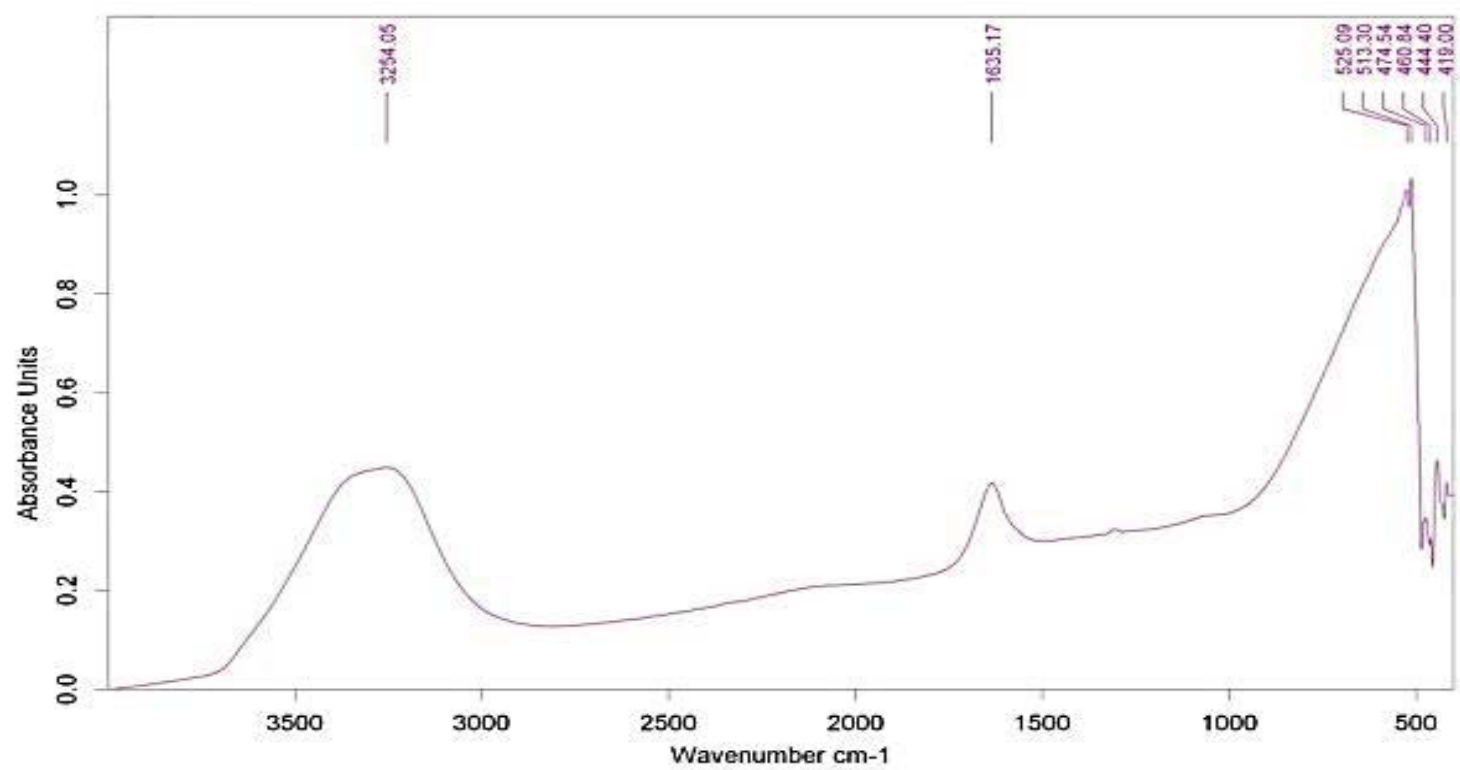

A.

B.

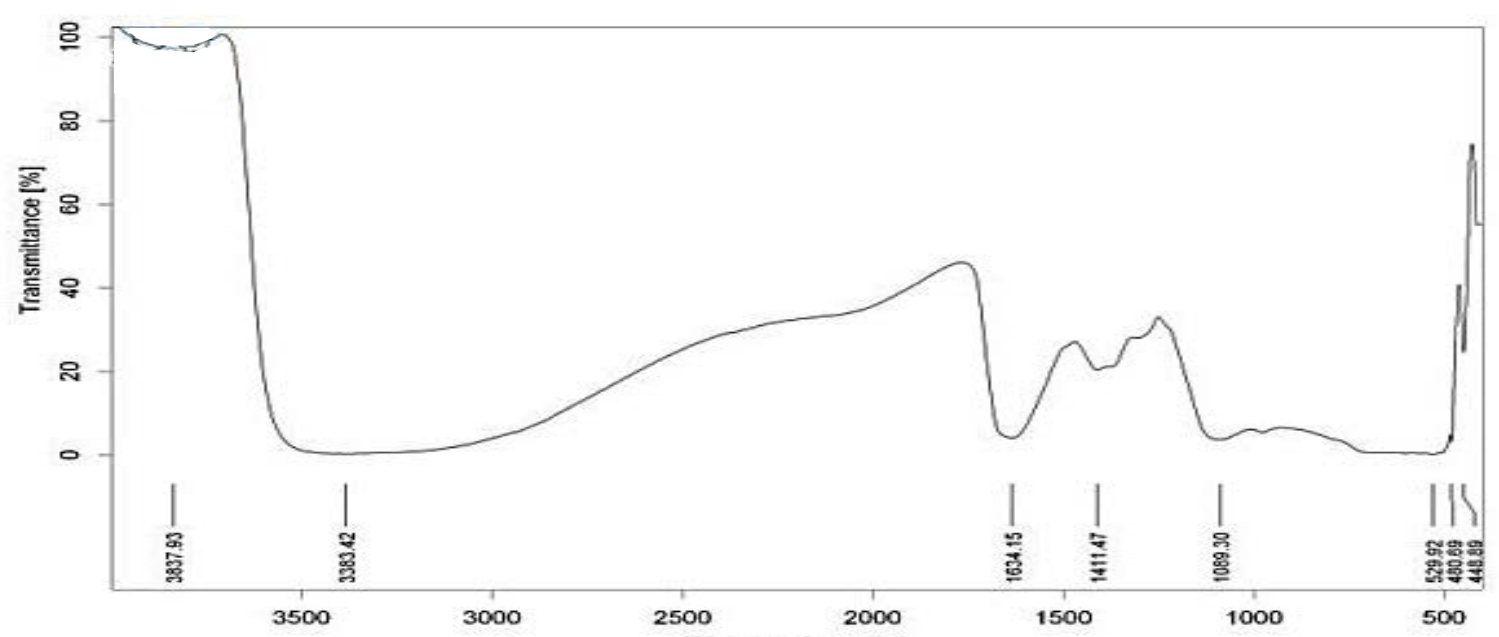

Fig. 6: FTIR spectra of $M$. ornata leaf extract and FeNPs synthesized using $M$. ornata flower sheath extract

(A) FTIR spectrum of M. ornata leaf extract and (B) FTIR spectrum of the FeNPs synthesized using M. ornata flower sheath extract. The presence of peak at $3254.05 \mathrm{~cm}^{-1}$ indicated the possible $\mathrm{O}-\mathrm{H}$ stretching vibration of phenol groups, which might be responsible for the formation and stabilization of nanoparticles

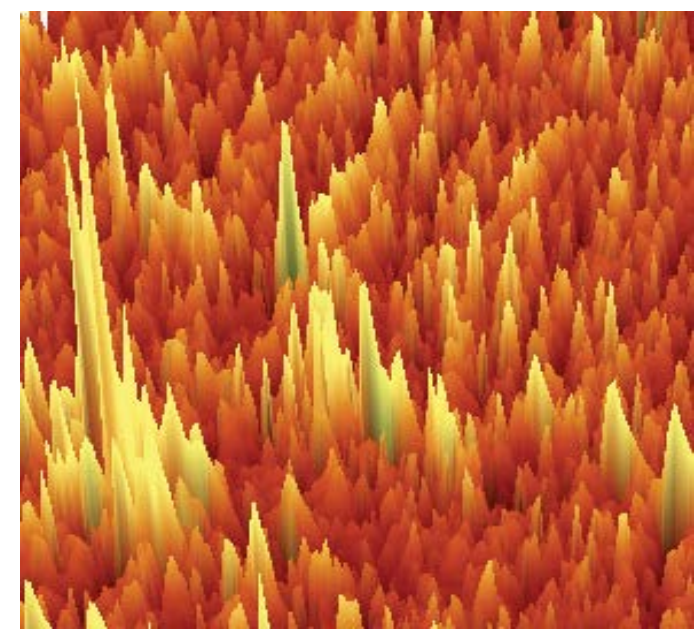

Fig. 7: Atomic force microscopic image of FeNPs

AFM topographic three dimensional images indicated the physically dispersed FeNPs

September-October 2017

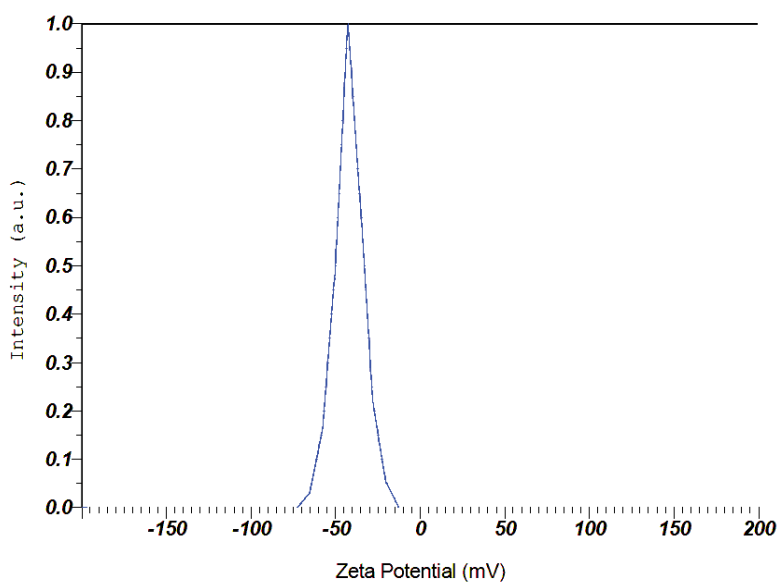

Fig. 8: Zeta potential analysis of FeNPs

The theoretical limit of stability of particles in a colloidal system is $30 \mathrm{mV}^{[18]}$ and iron nanoparticles synthesized, had a zeta potential value of $\pm 35 \mathrm{mV}$ indicating that the synthesized FeNPs had moderate stability 


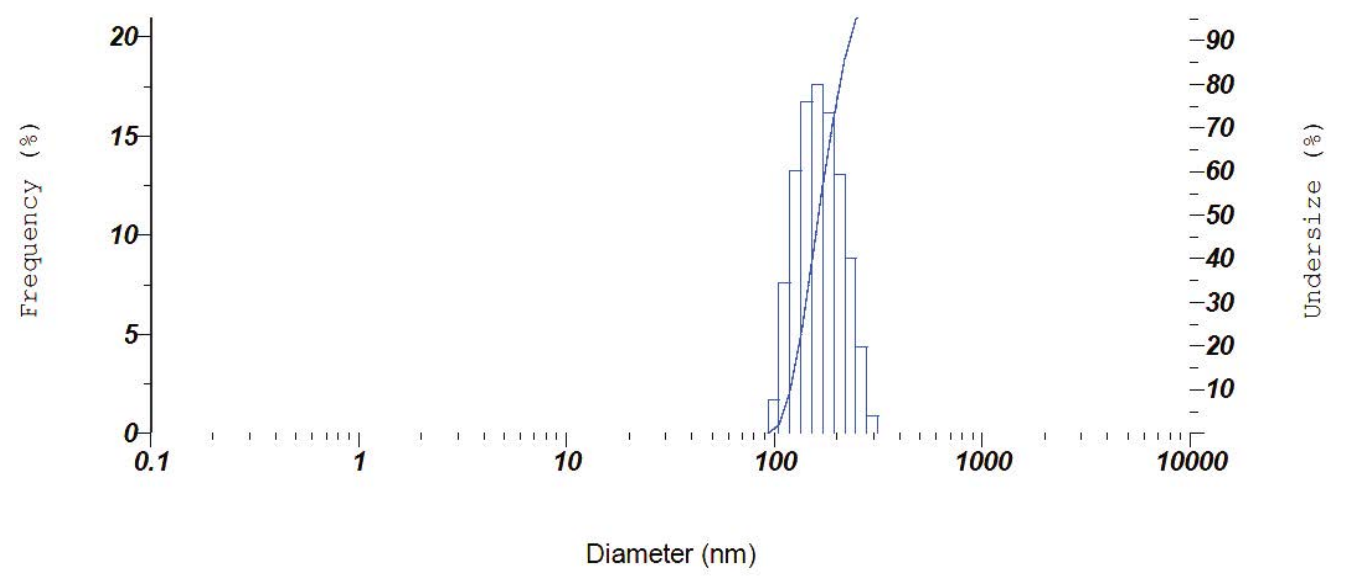

Fig. 9: Percent frequency of particle size distribution of FeNPs

The frequency distribution on Y-axis was on weight basis. The average particle size ( $\mathrm{z}$ - average) was found to be $86.2 \mathrm{~nm}$
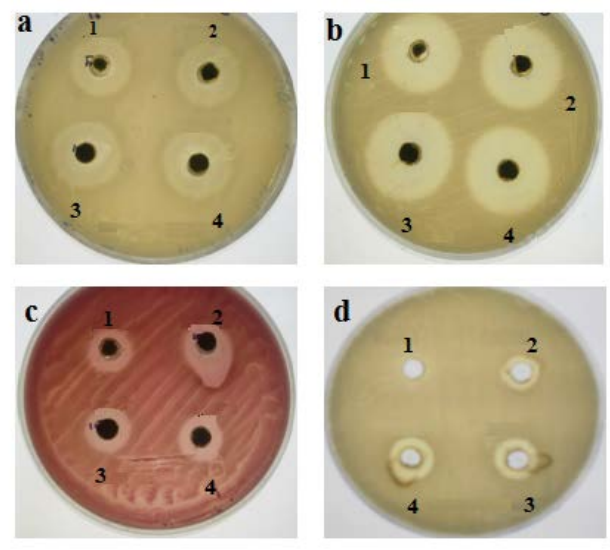

Fig. 10: Antibacterial activity of FeNPs against various bacteria (a) S. agalactiae in nutrient agar, (b) $S$. aureus in nutrient agar, (c) $E$. coli in MacConkey agar and (d) $S$. enterica in nutrient agar. (1) $5 \mathrm{mg} / \mathrm{ml}$; (2) $10 \mathrm{mg} / \mathrm{ml}$; (3) $15 \mathrm{mg} / \mathrm{ml}$; (4) $20 \mathrm{mg} / \mathrm{ml}$

as reducing and capping agents. The average crystal of FeNPs was found to be $43.69 \mathrm{~nm}$ and these nanoparticles had antibacterial activity.

\section{Acknowledgements:}

The authors thank the Government of India and Government of Tamil Nadu for supporting this work under the National Agricultural Development Programme (RKVY). The authors also thank the Director of Research and Dean, Faculty of Basic Sciences, Tamil Nadu Veterinary and Animal Sciences University, Dean, Madras Veterinary College, Chennai, Professor and Head, Department of Animal Biotechnology, Madras Veterinary College and the Professor and Head, University Research Farm, Madhavaram for the facilities provided.

\section{Conflict of interest:}

Nil.

\section{Financial support and sponsorship:}

Nil.

\section{REFERENCES}

1. Albrecht MA, Evans CW, Raston CL. Green chemistry and the health implications of nanoparticles. Green Chem 2006;8:417-32.

2. De D, Mandal MM, Gauri SS, Bhattacharya R, Ram S, Roy SK. Antibacterial effect of lanthanum calcium manganite (La0.67Ca0.33MnO3) nanoparticles against Pseudomonas aeruginosa ATCC 27853. J Biomed Nanotechnol 2010;6:138-44.

3. Dixon MB, Falconet C, Ho L, Christopher WK, O'Neill BK, Newcombe G. Removal of cyanobacterial metabolites by nano filtration from two treated waters. J Hazard Mater 2011;1882:88-95.

4. Sastry M, Ahmad A, Khan MI, Kumar R. Microbial nanoparticle production. In: Niemeyer CM, Mirkin CA, editors. Nanobiotechnology: Concepts, Applications and Perspectives. Weinheim: Wiley-VCH Verlag $\mathrm{GmbH}$ and Co. KGaA; 2004. p. 126-35.

5. Bhattacharya $D$, Rajinder G. Nanotechnology and potential of microorganisms. Crit Rev Biotechnol 2005;25:199-204.

6. Mohanpuria P, Rana NK, Yadav SK. Biosynthesis of nanoparticles: technological concepts and future applications. J Nano Part Res 2008;10:507-17.

7. Rosi NL, Giljohann DA, Thaxton, CS, Lytton-Jean AKR, Han MS, Mirkin CA. Oligonucleotide-modified gold nanoparticles for intracellular gene regulation. Science 2006;312:1027.

8. Shchukin DG, Schattka JH, Antonietti M, Caruso RA. Synthesis of nano-sized magnetic ferrite particles inside hollow polyelectrolyte capsules. J Phys Chem B 2003;107:952.

9. Shobha G, Vinutha M, Ananda S. Biological synthesis of copper nanoparticles and its impact - a Review. Int J Pharm Sci Invent 2014;38:28-38.

10. Sawai J. Quantitative evaluation of antibacterial activities of metallic oxide powders $(\mathrm{ZnO}, \mathrm{MgO}$ and $\mathrm{CaO})$ by conductimetric assay. J Microbiol Methods 2003;54:177-82.

11. Shahverdi AR, Fakhimi A, Shahverdi HR, Minaian S. Synthesis and effects of silver nanoparticles on the antibacterial activity of different antibiotics against Staphylococcus aureus and Escherichia coli. J Nanomedicine 2007;3:168-17. 
12. Pattanayak M, Nayak PL. Green synthesis and characterization of zero valent iron nanoparticles from the leaf extract of Azadirachta indica (neem). World J Nano Sci Tech 2013;2:6-9.

13. Sarkar B, Bhattacharjee S, Daware A, Tribedi P, Krishnan KK, Minhas PS. Selenium nanoparticles for stress resilient fish and livestock. Nanoscale Res Lett 2015;10:371.

14. Sathishkumar M, Sneha K, Won SW, Cho CW, Kim S, Yun YS. Cinnamon zeylanicum bark extract and powder mediated green synthesis of nano-crystalline silver particles and its bactericidal activity. Colloids Surf B Biointerfaces 2009; 73:332-8.

15. Latha N, Gowri M. Bio synthesis and characterization of
Fe3O4 nanoparticles using Caricaya papaya leaves extract. Int J Sci Res 2014;3:1551-6.

16. Kumar A, Singhal A. Synthesis of colloidal $\beta-\mathrm{Fe} 2 \mathrm{O} 3$ nanostructures-influence of addition of $\mathrm{Co}_{2}^{+}$on their morphology and magnetic behaviour. $\mathrm{J}$ Nanotech 2007; 18:475703.

17. Lee J, Isobe T, Senna M. Preparation of ultrafine Fe3O4 particles by precipitation in the presence of PVA at high $\mathrm{pH}$. J Colloid Interface Sci 1996;177:490.

18. Duman O, Tunc S. Electrokinetic and rheological properties of Na-bentonite in some electrolyte solutions. Micropor Mesopor Mat 2009;117:331-8. 